QUARTERLY OF APPLIED MATHEMATICS

VOLUME LXIII, NUMBER 2

JUNE 2005, PAGES 277-289

S 0033-569X(05)00947-5

Article electronically published on April 11, 2005

\title{
PERIODIC SOLUTION OF A NONAUTONOMOUS STAGE-STRUCTURED SINGLE SPECIES MODEL WITH DIFFUSION
}

\author{
BY \\ ZHENGQIU ZHANG (Department of Applied Mathematics, Hunan University, Changsha, 410082, \\ People's Republic of China) \\ AND \\ SHANWU ZENG (College of Mathematics, Wuhan University, Wuhan, 430072, People's Republic of \\ China)
}

Abstract. A stage-structured single species model with diffusion is considered in which the coefficients are time-dependent. By using the continuation theorem of coincidence degree theory, a sufficient condition is obtained for the existence of a positive periodic solution for this model.

1. Introduction. Recently the so-called single species model was considered and many results were obtained; see [1]-[6]. In [1], the stage-structured single species model, given by

$$
\begin{aligned}
& \dot{x}_{i}(t)=\alpha x_{m}(t)-\gamma x_{i}(t)-\alpha e^{-\gamma \tau} \phi(t-\tau), \\
& \dot{x}_{m}(t)=\alpha e^{-\gamma \tau} \phi(t-\tau)-\beta x_{m}^{2}(t), \quad 0<t \leq \tau, \\
& \dot{x}_{i}(t)=\alpha x_{m}(t)-\gamma x_{i}(t)-\alpha e^{-\gamma \tau} x_{m}(t-\tau), \\
& \dot{x}_{m}(t)=\alpha e^{-\gamma \tau} x_{m}(t-\tau)-\beta x_{m}^{2}(t), \quad t>\tau,
\end{aligned}
$$

was studied, where $x_{i}(t)$ and $x_{m}(t)$ represent the immature and mature population densities, respectively; $\phi$ is the observed or assumed birth rate of $x_{i}(t)$ at time $t(-\tau \leq t \leq 0)$, and $\tau$ represents a constant time length for the immature to become mature. $\alpha$ is the birth rate of mature population. $\gamma$ is the death rate of the immature population and $\beta$ is the preventing function caused by loss of limited resource of the mature population. Some sufficient conditions for the stability of the positive equilibrium were also given.

In [4, the nonautonomous single species models with diffusion were considered and the following model was proposed:

$$
\left\{\begin{array}{l}
\dot{x}_{1}=x_{1}\left(r_{1}(t)-a_{11} x_{1}\right)+D_{1}(t)\left(x_{2}-x_{1}\right) \\
\dot{x}_{2}=x_{2}\left(r_{2}(t)-a_{22} x_{2}\right)+D_{2}(t)\left(x_{1}-x_{2}\right)
\end{array}\right.
$$

Received October 16, 2003.

2000 Mathematics Subject Classification. Primary 34C25.

Key words and phrases. Nonautonomous, stage-structured, single species, diffusion, periodic solution, the continuation theorem of coincidence degree, topological degree.

Project supported by NNSF of China (No. 10271044).

E-mail address: z-q-zhang@sina.com 
Species $x$ grows in both patch 1 and patch 2 ; i.e., species $x$ can diffuse between patch 1 and patch 2 , where $x_{1}(t)$ is the density of the species in patch 1 at time $t(t \geq 0)$ and $x_{2}(t)$ denotes the density in patch 2. $D_{1}(t)$ and $D_{2}(t)$ are diffusion coefficients. In [4], criteria for the existence and uniqueness of periodic solutions of these systems were established.

However, for a single species which has both stage structure and the ability of diffusion, there is no proper model studied as yet. For many species, their rate of death and the ability to diffuse are greatly different in each stage of their life stories. For example, the immature red-crowned crane is unable to migrate until it reaches a certain life stage, while in its mature stage, it is able to migrate. As another example, some fish have much higher death rates in their immature stage than in their mature stage. Hence it is not proper to take for granted that a given species has the same death rate in every stage of its life. Therefore the models proposed in [4]-[6], in which stage-structures were ignored, are not necessarily correct.

On the other hand, in papers [1]-[3], the diffusion is ignored and all the coefficients are constants; that is, the environments do not vary with time. Obviously those populations with time-dependence and diffusion cannot be described by these models. Hence it is significant for us to study stage-structured nonautonomous single species models with diffusion.

In this paper, we assume that the single species populations are growing in a two-patch environment, each of which is closed and homogeneous. Let $x_{1}(t)$ and $x_{2}(t)$ denote the densities of immature and mature populations in patch 1 . Let $y_{1}(t)$ and $y_{2}(t)$ denote the densities of the immature and mature populations in patch 2 . Now, our model is described by the following system:

$$
\left\{\begin{array}{l}
\dot{x}_{1}=b_{1}(t) x_{2}-g_{1}(t) x_{1}-d_{1}(t) x_{1}^{2}, \\
\dot{x}_{2}=g_{1}(t) x_{1}-D_{1}(t) x_{2}^{2}+\lambda_{1}(t)\left(y_{2}-x_{2}\right), \\
\dot{y}_{1}=b_{2}(t) y_{2}-g_{2}(t) y_{1}-d_{2}(t) y_{1}^{2}, \\
\dot{y}_{2}=g_{2}(t) y_{1}-D_{2}(t) y_{2}^{2}+\lambda_{2}(t)\left(x_{2}-y_{2}\right),
\end{array}\right.
$$

where $b_{1}(t)$ is the birth rate of the immature population in patch 1 and $b_{2}(t)$ is the birth rate in patch $2 ; g_{1}(t)$ and $g_{2}(t)$ denote the transformation rates of the immature populations into the mature in patch 1 and patch 2, respectively. $d_{1}(t)$ and $d_{2}(t)$ are the density-dependent coefficients of the immature population in patch 1 and 2, respectively, and $D_{1}(t)$ and $D_{2}(t)$ are the same for the mature population in the corresponding patch. $\lambda_{1}(t)$ and $\lambda_{2}(t)$ are diffusion coefficients. We assume that all the coefficients in the model are strictly positive continuous $\omega$-periodic functions.

Our purpose in this paper is, by using Mawhin's continuation theorem [7, 8], to study the existence of positive $\omega$-periodic solutions for system (1.1). For work concerning the existence of periodic solutions of delay differential equations, which was done using coincidence degree theory, we refer to [9], [10] and references cited therein. 
2. Existence of a positive periodic solution. In this section, by using Mawhin's continuation theorem, we shall show the existence of at least one positive periodic solution of system (1.1). To do so, we need to make some preparations.

Let $X$ and $Y$ be real Banach spaces, let $L: \operatorname{Dom} L \subset X \rightarrow Y$ be a Fredholm mapping of index zero, and let $P: X \rightarrow X, Q: Y \rightarrow Y$ be continuous projectors such that $\operatorname{Im} P=\operatorname{Ker} L, \operatorname{Ker} Q=\operatorname{Im} L$, and $X=\operatorname{Ker} L \oplus \operatorname{Ker} P, Y=\operatorname{Im} L \oplus \operatorname{Im} Q$. Denote by $L_{p}$ the restriction of $L$ to $\operatorname{Dom} L \cap \operatorname{Ker} P, K_{p}: \operatorname{Im} L \rightarrow \operatorname{Ker} P \cap \operatorname{Dom} L$ the inverse (to $L_{p}$ ), and $J: \operatorname{Im} Q \rightarrow \operatorname{Ker} L$ an isomorphism of $\operatorname{Im} Q$ onto $\operatorname{Ker} L$.

For convenience, we introduce Mawhin's continuation theorem [7, p. 40] as follows.

Lemma 2.1. Let $\Omega \subset X$ be an open bounded set and let $N: X \rightarrow Y$ be a continuous operator which is $L$-compact on $\bar{\Omega}$ (i.e., $Q N: \bar{\Omega} \rightarrow Y$ and $K_{p}(I-Q) N: \bar{\Omega} \rightarrow Y$ are compact). Assume

(i) for each $\lambda \in(0,1), x \in \partial \Omega \cap \operatorname{Dom} L, L x \neq \lambda N x$;

(ii) for each $x \in \partial \Omega \cap \operatorname{Ker} L, Q N x \neq 0$;

(iii) $\operatorname{deg}\{J Q N, \Omega \cap \operatorname{Ker} L, 0\} \neq 0$.

Then $L x=N x$ has at least one solution in $\bar{\Omega} \cap \operatorname{Dom} L$.

In what follows, we use the notation

$$
\bar{f}=\frac{1}{\omega} \int_{0}^{\omega} f(t) d t, \quad f^{l}=\min _{t \in[0, \omega]}|f(t)|, \quad f^{u}=\max _{t \in[0, \omega]}|f(t)|,
$$

where $f$ is a periodic continuous function with period $\omega>0$.

In system (1.1), we always assume the following.

Assumption $(\mathrm{H} 1) . b_{i}(t), g_{i}(t), d_{i}(t), D_{i}(t), \lambda_{i}(t)(i=1,2)$ are positive periodic continuous functions with periodic $\omega>0$.

Now we state our fundamental theorem about the existence of a positive $\omega$-periodic solution of system (1.1).

Theorem 2.1. In addition to Assumption (H1), we assume

(H2) $g_{1}^{l} b_{1}^{l}>g_{1}^{u} \lambda_{1}^{u}$

(H3) $g_{2}^{l} b_{2}^{l}>g_{2}^{u} \lambda_{2}^{u}$.

Then system (1.1) has at least one positive $\omega$-periodic solution.

Proof. Consider the system

$$
\left\{\begin{array}{l}
\frac{d u_{1}(t)}{d t}=b_{1}(t) e^{u_{2}(t)-u_{1}(t)}-g_{1}(t)-d_{1}(t) e^{u_{1}(t)} \\
\frac{d u_{2}(t)}{d t}=g_{1}(t) e^{u_{1}(t)-u_{2}(t)}-D_{1}(t) e^{u_{2}(t)}+\lambda_{1}(t)\left(e^{u_{4}(t)-u_{2}(t)}-1\right), \\
\frac{d u_{3}(t)}{d t}=b_{2}(t) e^{u_{4}(t)-u_{3}(t)}-g_{2}(t)-d_{2}(t) e^{u_{3}(t)} \\
\frac{d u_{4}(t)}{d t}=g_{2}(t) e^{u_{3}(t)-u_{4}(t)}-D_{2}(t) e^{u_{4}(t)}+\lambda_{2}(t)\left(e^{u_{2}(t)-u_{4}(t)}-1\right),
\end{array}\right.
$$

where $b_{i}(t), g_{i}(t), d_{i}(t), D_{i}(t), \lambda_{i}(t)(i=1,2)$ are the same as those in Assumption (H1). Let $x_{i}(t)=e^{u_{i}(t)}, i=1,2, y_{1}(t)=e^{u_{3}(t)}, y_{2}(t)=e^{u_{4}(t)}$. Then system (1.1) changes into system (2.1). Hence it is easy to see that if system (2.1) has an $\omega$-periodic solution $\left(u_{1}^{*}(t), u_{2}^{*}(t), u_{3}^{*}(t), u_{4}^{*}(t)\right)^{T}$, then $\left(e^{u_{1}^{*}(t)}, e^{u_{2}^{*}(t)}, e^{u_{3}^{*}(t)}, e^{u_{4}^{*}(t)}\right)^{T}$ is a positive $\omega$-periodic solution of system (1.1). Therefore, for (1.1) to have at least one positive $\omega$-periodic solution, it is sufficient that (2.1) have at least one $\omega$-periodic solution. In order to apply 
Lemma 2.1 to system (2.1), we take

$$
X=Y=\left\{\left(u_{1}(t), u_{2}(t), u_{3}(t), u_{4}(t)\right)^{T} \in C\left(R, R^{4}\right): u_{i}(t+\omega)=u_{i}(t), i=1,2,3,4\right\}
$$

and

$\left\|\left(u_{1}(t), u_{2}(t), u_{3}(t), u_{4}(t)\right)^{T}\right\|=\max _{t \in[0, \omega]}\left|u_{1}(t)\right|+\max _{t \in[0, \omega]}\left|u_{2}(t)\right|+\max _{t \in[0, \omega]}\left|u_{3}(t)\right|+\max _{t \in[0, \omega]}\left|u_{4}(t)\right|$,

where $|\cdot|$ denotes the Euclidean norm in $R$. With the norm $\|\cdot\|, X$ is a Banach space. Set $L: D o m L \cap X, L\left(u_{1}(t), u_{2}(t), u_{3}(t), u_{4}(t)\right)^{T}=\left(u_{1}^{\prime}(t), u_{2}^{\prime}(t), u_{3}^{\prime}(t), u_{4}^{\prime}(t)\right)^{T}$, where $D o m L=\left\{\left(u_{1}(t), u_{2}(t), u_{3}(t), u_{4}(t)\right)^{T} \in C^{1}\left(R, R^{4}\right)\right\}, N: X \rightarrow X$, and

$$
N\left[\begin{array}{l}
u_{1} \\
u_{2} \\
u_{3} \\
u_{4}
\end{array}\right]=\left[\begin{array}{c}
b_{1}(t) e^{u_{2}(t)-u_{1}(t)}-g_{1}(t)-d_{1}(t) e^{u_{1}(t)} \\
g_{1}(t) e^{u_{1}(t)-u_{2}(t)}-D_{1}(t) e^{u_{2}(t)}+\lambda_{1}(t)\left(e^{u_{4}(t)-u_{2}(t)}-1\right) \\
b_{2}(t) e^{u_{4}(t)-u_{3}(t)}-g_{2}(t)-d_{2}(t) e^{u_{3}(t)} \\
g_{2}(t) e^{u_{3}(t)-u_{4}(t)}-D_{2}(t) e^{u_{4}(t)}+\lambda_{2}(t)\left(e^{u_{2}(t)-u_{4}(t)}-1\right)
\end{array}\right] .
$$

Define two projectors $P$ and $Q$ as follows:

$$
P\left[\begin{array}{l}
u_{1} \\
u_{2} \\
u_{3} \\
u_{4}
\end{array}\right]=Q\left[\begin{array}{l}
u_{1} \\
u_{2} \\
u_{3} \\
u_{4}
\end{array}\right]=\left[\begin{array}{l}
\frac{1}{\omega} \int_{0}^{\omega} u_{1}(t) d t \\
\frac{1}{\omega} \int_{0}^{\omega} u_{2}(t) d t \\
\frac{1}{\omega} \int_{0}^{\omega} u_{3}(t) d t \\
\frac{1}{\omega} \int_{0}^{\omega} u_{4}(t) d t
\end{array}\right], \quad\left[\begin{array}{l}
u_{1} \\
u_{2} \\
u_{3} \\
u_{4}
\end{array}\right] \in X .
$$

Clearly, $\operatorname{Ker} L=R^{4}, \operatorname{ImL}=\left\{\left(u_{1}, u_{2}, u_{3}, u_{4}\right)^{T} \in X: \int_{0}^{\omega} u_{i}(t) d t=0, i=1,2,3,4\right\}$ is closed in $X$ and $\operatorname{dimKer} L=\operatorname{codim} I m L=4$. Hence $L$ is a Fredholm mapping of index zero. Furthermore, through an easy computation, we can find that the inverse $K_{p}$ of $L_{p}$ has the form $K_{p}: \operatorname{ImL} \rightarrow \operatorname{Dom} L \cap \operatorname{KerP}$,

$$
K_{p}\left[\begin{array}{l}
u_{1} \\
u_{2} \\
u_{3} \\
u_{4}
\end{array}\right]=\left[\begin{array}{l}
\int_{0}^{t} u_{1}(s) d s-\frac{1}{\omega} \int_{0}^{\omega} \int_{0}^{\eta} u_{1}(s) d t d \eta \\
\int_{0}^{t} u_{2}(s) d s-\frac{1}{\omega} \int_{0}^{\omega} \int_{0}^{\eta} u_{2}(s) d t d \eta \\
\int_{0}^{t} u_{3}(s) d s-\frac{1}{\omega} \int_{0}^{\omega} \int_{0}^{\eta} u_{3}(s) d t d \eta \\
\int_{0}^{t} u_{4}(s) d s-\frac{1}{\omega} \int_{0}^{\omega} \int_{0}^{\eta} u_{4}(s) d t d \eta
\end{array}\right]
$$

Obviously, we can prove that $Q N$ and $K_{p}(I-Q) N$ are continuous by Lebesgue's theorem and that $Q N(\bar{\Omega}), K_{p}(I-Q) N(\bar{\Omega})$ are relatively compact for any open bounded set $\Omega \subset X$ by the Arzela-Ascoli theorem. Therefore, $N$ is $L$-compact on $\bar{\Omega}$ for any open bounded set $\Omega \subset X$. Corresponding to the operator equation $L x=\lambda N x, \lambda \in(0,1)$, we have

$$
\left\{\begin{array}{l}
\frac{d u_{1}(t)}{d t}=\lambda\left[b_{1}(t) e^{u_{2}(t)-u_{1}(t)}-g_{1}(t)-d_{1}(t) e^{u_{1}(t)}\right], \\
\frac{d u_{2}(t)}{d t}=\lambda\left[g_{1}(t) e^{u_{1}(t)-u_{2}(t)}-D_{1}(t) e^{u_{2}(t)}+\lambda_{1}(t) e^{u_{4}(t)-u_{2}(t)}-\lambda_{1}(t)\right], \\
\frac{d u_{3}(t)}{d t}=\lambda\left[b_{2}(t) e^{u_{4}(t)-u_{3}(t)}-g_{2}(t)-d_{2}(t) e^{u_{3}(t)}\right], \\
\frac{d u_{4}(t)}{d t}=\lambda\left[g_{2}(t) e^{u_{3}(t)-u_{4}(t)}-D_{2}(t) e^{u_{4}(t)}+\lambda_{2}(t) e^{u_{2}(t)-u_{4}(t)}-\lambda_{2}(t)\right] .
\end{array}\right.
$$


Suppose that $\left(u_{1}(t), u_{2}(t), u_{3}(t), u_{4}(t)\right)^{T} \in X$ is a solution of system (2.2) for a certain $\lambda \in(0,1)$. Integrating (2.2) from 0 to $\omega$ gives

$$
\begin{gathered}
\int_{0}^{\omega} g_{1}(t) e^{u_{1}(t)-u_{2}(t)} d t+\int_{0}^{\omega} \lambda_{1}(t) e^{u_{4}(t)-u_{2}(t)} d t=\int_{0}^{\omega} D_{1}(t) e^{u_{2}(t)} d t+\int_{0}^{\omega} \lambda_{1}(t) d t, \\
\int_{0}^{\omega} b_{2}(t) e^{u_{4}(t)-u_{3}(t)} d t=\int_{0}^{\omega} g_{2}(t) d t+\int_{0}^{\omega} d_{2}(t) e^{u_{3}(t)} d t
\end{gathered}
$$

and

$$
\int_{0}^{\omega} g_{2}(t) e^{u_{3}(t)-u_{4}(t)} d t+\int_{0}^{\omega} \lambda_{2}(t) e^{u_{2}(t)-u_{4}(t)} d t=\int_{0}^{\omega} D_{2}(t) e^{u_{4}(t)} d t+\int_{0}^{\omega} \lambda_{2}(t) d t .
$$

From (2.2)-(2.6), it follows that

$$
\begin{aligned}
& \int_{0}^{\omega}\left|u_{1}^{\prime}(t)\right| d t \leq \int_{0}^{\omega} b_{1}(t) e^{u_{2}(t)-u_{1}(t)} d t+\int_{0}^{\omega} g_{1}(t) d t+\int_{0}^{\omega} d_{1}(t) e^{u_{1}(t)} d t \\
& =2 \bar{g}_{1} \omega+2 \int_{0}^{\omega} d_{1}(t) e^{u_{1}(t)} d t \\
& \leq 2 \bar{g}_{1} \omega+2 d_{1}^{u} \int_{0}^{\omega} e^{u_{1}(t)} d t, \\
& \int_{0}^{\omega}\left|u_{2}^{\prime}(t)\right| d t \\
& \leq \int_{0}^{\omega} g_{1}(t) e^{u_{1}(t)-u_{2}(t)} d t+\int_{0}^{\omega} D_{1}(t) e^{u_{2}(t)} d t+\int_{0}^{\omega} \lambda_{1}(t) e^{u_{4}(t)-u_{2}(t)} d t+\int_{0}^{\omega} \lambda_{1}(t) d t \\
& =2 \bar{\lambda}_{1} \omega+2 \int_{0}^{\omega} D_{1}(t) e^{u_{2}(t)} d t \\
& \leq 2 \bar{\lambda}_{1} \omega+2 D_{1}^{u} \int_{0}^{\omega} e^{u_{2}(t)} d t,
\end{aligned}
$$

$$
\begin{gathered}
\int_{0}^{\omega}\left|u_{3}^{\prime}(t)\right| d t \leq \int_{0}^{\omega} b_{2}(t) e^{u_{4}(t)-u_{3}(t)} d t+\int_{0}^{\omega} g_{2}(t) d t+\int_{0}^{\omega} d_{2}(t) e^{u_{3}(t)} d t \\
=2 \bar{g}_{2} \omega+2 \int_{0}^{\omega} d_{2}(t) e^{u_{3}(t)} d t \\
\leq 2 \bar{g}_{2} \omega+2 d_{2}^{u} \int_{0}^{\omega} e^{u_{3}(t)} d t
\end{gathered}
$$


and

$$
\begin{aligned}
\int_{0}^{\omega} & \left|u_{4}^{\prime}(t)\right| d t \\
& \leq \int_{0}^{\omega} g_{2}(t) e^{u_{3}(t)-u_{4}(t)} d t+\int_{0}^{\omega} D_{2}(t) e^{u_{4}(t)} d t+\int_{0}^{\omega} \lambda_{2}(t) e^{u_{2}(t)-u_{4}(t)} d t+\int_{0}^{\omega} \lambda_{2}(t) d t \\
& =2 \bar{\lambda}_{2} \omega+2 \int_{0}^{\omega} D_{2}(t) e^{u_{4}(t)} d t \\
& \leq 2 \bar{\lambda}_{2} \omega+2 D_{2}^{u} \int_{0}^{\omega} e^{u_{4}(t)} d t
\end{aligned}
$$

From (2.2), we get

$$
\int_{0}^{\omega} d_{1}(t) e^{2 u_{1}(t)} d t+\int_{0}^{\omega} g_{1}(t) e^{u_{1}(t)} d t=\int_{0}^{\omega} b_{1}(t) e^{u_{2}(t)} d t
$$

$$
\begin{gathered}
\int_{0}^{\omega} D_{1}(t) e^{2 u_{2}(t)} d t+\int_{0}^{\omega} \lambda_{1}(t) e^{u_{2}(t)} d t=\int_{0}^{\omega} g_{1}(t) e^{u_{1}(t)} d t+\int_{0}^{\omega} \lambda_{1}(t) e^{u_{4}(t)} d t \\
\int_{0}^{\omega} d_{2}(t) e^{2 u_{3}(t)} d t+\int_{0}^{\omega} g_{2}(t) e^{u_{3}(t)} d t=\int_{0}^{\omega} b_{2}(t) e^{u_{4}(t)} d t
\end{gathered}
$$

and

$$
\int_{0}^{\omega} D_{2}(t) e^{2 u_{4}(t)} d t+\int_{0}^{\omega} \lambda_{2}(t) e^{u_{4}(t)} d t=\int_{0}^{\omega} g_{2}(t) e^{u_{3}(t)} d t+\int_{0}^{\omega} \lambda_{2}(t) e^{u_{2}(t)} d t .
$$

By using the inequality

$$
\int_{0}^{\omega} e^{2 u_{i}(t)} d t \geq \frac{1}{\omega}\left(\int_{0}^{\omega} e^{u_{i}(t)} d t\right)^{2}, \text { for } i=1,2,3,4,
$$

from (2.11)-(2.14), we obtain

$$
\begin{gathered}
\frac{d_{1}^{l}}{\omega}\left(\int_{0}^{\omega} e^{u_{1}(t)} d t\right)^{2}+g_{1}^{l} \int_{0}^{\omega} e^{u_{1}(t)} d t \leq b_{1}^{u} \int_{0}^{\omega} e^{u_{2}(t)} d t \\
\frac{D_{1}^{l}}{\omega}\left(\int_{0}^{\omega} e^{u_{2}(t)} d t\right)^{2}+\lambda_{1}^{l} \int_{0}^{\omega} e^{u_{2}(t)} d t \leq g_{1}^{u} \int_{0}^{\omega} e^{u_{1}(t)} d t+\lambda_{1}^{u} \int_{0}^{\omega} e^{u_{4}(t)} d t \\
\frac{d_{2}^{l}}{\omega}\left(\int_{0}^{\omega} e^{u_{3}(t)} d t\right)^{2}+g_{2}^{l} \int_{0}^{\omega} e^{u_{3}(t)} d t \leq b_{2}^{u} \int_{0}^{\omega} e^{u_{4}(t)} d t
\end{gathered}
$$

and

$$
\frac{D_{2}^{l}}{\omega}\left(\int_{0}^{\omega} e^{u_{4}(t)} d t\right)^{2}+\lambda_{2}^{l} \int_{0}^{\omega} e^{u_{4}(t)} d t \leq g_{2}^{u} \int_{0}^{\omega} e^{u_{3}(t)} d t+\lambda_{2}^{u} \int_{0}^{\omega} e^{u_{2}(t)} d t .
$$

Inequality (2.15) gives

$$
\frac{d_{1}^{l}}{\omega}\left(\int_{0}^{\omega} e^{u_{1}(t)} d t\right)^{2}<b_{1}^{u} \int_{0}^{\omega} e^{u_{2}(t)} d t
$$


that is,

$$
\int_{0}^{\omega} e^{u_{1}(t)} d t<\sqrt{\frac{b_{1}^{u} \omega}{d_{1}^{l}}}\left(\int_{0}^{\omega} e^{u_{2}(t)} d t\right)^{\frac{1}{2}}
$$

Substituting (2.19) into (2.16) gives

$$
\frac{D_{1}^{l}}{\omega}\left(\int_{0}^{\omega} e^{u_{2}(t)} d t\right)^{2}+\lambda_{1}^{l} \int_{0}^{\omega} e^{u_{2}(t)} d t<g_{1}^{u} \sqrt{\frac{b_{1}^{u} \omega}{d_{1}^{l}}}\left(\int_{0}^{\omega} e^{u_{2}(t)} d t\right)^{\frac{1}{2}}+\lambda_{1}^{u} \int_{0}^{\omega} e^{u_{4}(t)} d t,
$$

from which, by using the inequality

$$
1 \cdot\left(\int_{0}^{\omega} e^{u_{2}(t)} d t\right)^{\frac{1}{2}} \leq \frac{1}{2}+\frac{\int_{0}^{\omega} e^{u_{2}(t)} d t}{2}
$$

we get

$$
\frac{D_{1}^{l}}{\omega}\left(\int_{0}^{\omega} e^{u_{2}(t)} d t\right)^{2}<\frac{g_{1}^{u}}{2} \sqrt{\frac{b_{1}^{u} \omega}{d_{1}^{l}}} \int_{0}^{\omega} e^{u_{2}(t)} d t+\lambda_{1}^{u} \int_{0}^{\omega} e^{u_{4}(t)} d t+\frac{g_{1}^{u}}{2} \sqrt{\frac{b_{1}^{u} \omega}{d_{1}^{l}}} .
$$

Then

$$
\frac{2 D_{1}^{l}}{\omega} \int_{0}^{\omega} e^{u_{2}(t)} d t<\frac{g_{1}^{u}}{2} \sqrt{\frac{b_{1}^{u} \omega}{d_{1}^{l}}}+\left\{\frac{\left(g_{1}^{u}\right)^{2} b_{1}^{u} \omega}{4 d_{1}^{l}}+\frac{4 D_{1}^{l}}{\omega} \lambda_{1}^{u} \int_{0}^{\omega} e^{u_{4}(t)} d t+\frac{2 D_{1}^{l} g_{1}^{u}}{\omega} \sqrt{\frac{b_{1}^{u} \omega}{d_{1}^{l}}}\right\}^{\frac{1}{2}} .
$$

By using the inequality

$$
(a+b)^{\frac{1}{2}}<a^{\frac{1}{2}}+b^{\frac{1}{2}}, \text { for } a \geq 0, b \geq 0,
$$

and from (2.21), we obtain

$$
\frac{2 D_{1}^{l}}{\omega} \int_{0}^{\omega} e^{u_{2}(t)} d t<g_{1}^{u} \sqrt{\frac{b_{1}^{u} \omega}{d_{1}^{l}}}+2 \sqrt{\frac{D_{1}^{l} \lambda_{1}^{u}}{\omega}}\left(\int_{0}^{\omega} e^{u_{4}(t)} d t\right)^{\frac{1}{2}}+\sqrt{\frac{2 D_{1}^{l} g_{1}^{u}}{\omega}} \sqrt[4]{\frac{b_{1}^{u} \omega}{d_{1}^{l}}} .
$$

Therefore there exists a positive constant $d_{3}$ such that

$$
\int_{0}^{\omega} e^{u_{2}(t)} d t<d_{3}+\frac{\omega}{D_{1}^{l}} \sqrt{\frac{D_{1}^{l} \lambda_{1}^{u}}{\omega}}\left(\int_{0}^{\omega} e^{u_{4}(t)} d t\right)^{\frac{1}{2}} .
$$

From (2.17) and (2.18), a parallel argument to (2.23) shows that

$$
\int_{0}^{\omega} e^{u_{4}(t)} d t<d_{4}+\frac{\omega}{D_{2}^{l}} \sqrt{\frac{D_{2}^{l} \lambda_{2}^{u}}{\omega}}\left(\int_{0}^{\omega} e^{u_{2}(t)} d t\right)^{\frac{1}{2}}
$$

where $d_{4}$ is a positive constant.

Substituting (2.24) into (2.23) gives

$$
\int_{0}^{\omega} e^{u_{2}(t)} d t<d_{3}+\frac{\omega}{D_{1}^{l}} \sqrt{\frac{D_{1}^{l} \lambda_{1}^{u}}{\omega}}\left[d_{4}+\frac{\omega}{D_{2}^{l}} \sqrt{\frac{D_{2}^{l} \lambda_{2}^{u}}{\omega}}\left(\int_{0}^{\omega} e^{u_{2}(t)} d t\right)^{\frac{1}{2}}\right]^{\frac{1}{2}}
$$


from which, by using inequality (2.22), we obtain

$$
\begin{gathered}
\int_{0}^{\omega} e^{u_{2}(t)} d t<d_{3}+\frac{\omega}{D_{1}^{l}} \sqrt{\frac{D_{1}^{l} \lambda_{1}^{u}}{\omega}}\left[\sqrt{d_{4}}+\sqrt{\frac{\omega}{D_{2}^{l}}} \sqrt[4]{\frac{D_{2}^{l} \lambda_{2}^{u}}{\omega}}\left(\int_{0}^{\omega} e^{u_{2}(t)} d t\right)^{\frac{1}{4}}\right] \\
=d_{3}+\frac{\omega}{D_{1}^{l}} \sqrt{\frac{D_{1}^{l} \lambda_{1}^{u} d_{4}}{\omega}}+\frac{\omega}{D_{1}^{l}} \sqrt{\frac{D_{1}^{l} \lambda_{1}^{u}}{D_{2}^{l}}} \sqrt[4]{\frac{D_{2}^{l} \lambda_{2}^{u}}{\omega}}\left(\int_{0}^{\omega} e^{u_{2}(t)} d t\right)^{\frac{1}{4}} .
\end{gathered}
$$

Thus there exists a positive constant $\rho_{2}$ such that

$$
\int_{0}^{\omega} e^{u_{2}(t)} d t<\rho_{2} .
$$

Substituting (2.25) into (2.24), it follows that there exists a positive constant $\rho_{4}$ such that

$$
\int_{0}^{\omega} e^{u_{4}(t)} d t<\rho_{4} .
$$

From $(2.17),(2.19),(2.25)$ and $(2.26)$, there must exist two positive constants $\rho_{1}$ and $\rho_{3}$ such that

$$
\int_{0}^{\omega} e^{u_{1}(t)} d t<\rho_{1}, \quad \int_{0}^{\omega} e^{u_{3}(t)} d t<\rho_{3} .
$$

Thus from (2.7)-(2.10), (2.25)-(2.27), we obtain

$$
\begin{aligned}
& \int_{0}^{\omega}\left|u_{1}^{\prime}(t)\right| d t \leq 2 \bar{g}_{1} \omega+2 d_{1}^{u} \rho_{1} \stackrel{\text { def }}{=} \delta_{1}, \\
& \int_{0}^{\omega}\left|u_{2}^{\prime}(t)\right| d t \leq 2 \bar{\lambda}_{1} \omega+2 D_{1}^{u} \rho_{2} \stackrel{\text { def }}{=} \delta_{2}, \\
& \int_{0}^{\omega}\left|u_{3}^{\prime}(t)\right| d t \leq 2 \bar{g}_{2} \omega+2 d_{2}^{u} \rho_{3} \stackrel{\text { def }}{=} \delta_{3},
\end{aligned}
$$

and

$$
\int_{0}^{\omega}\left|u_{4}^{\prime}(t)\right| d t \leq 2 \bar{\lambda}_{2} \omega+2 D_{2}^{u} \rho_{4} \stackrel{\text { def }}{=} \delta_{4} .
$$

Choose $t_{i} \in[0, \omega], i=1,2,3,4$, such that

$$
u_{i}\left(t_{i}\right)=\min _{t \in[0, \omega]} u_{i}(t), \quad i=1,2,3,4 .
$$

Then it is clear that

$$
u_{i}^{\prime}\left(t_{i}\right)=0, \quad i=1,2,3 .
$$

From this and (2.2), we have

$$
\begin{gathered}
b_{1}\left(t_{1}\right) e^{u_{2}\left(t_{1}\right)-u_{1}\left(t_{1}\right)}-g_{1}\left(t_{1}\right)-d_{1}\left(t_{1}\right) e^{u_{1}\left(t_{1}\right)}=0, \\
g_{1}\left(t_{2}\right) e^{u_{1}\left(t_{2}\right)-u_{2}\left(t_{2}\right)}-D_{1}\left(t_{2}\right) e^{u_{2}\left(t_{2}\right)}+\lambda_{1}\left(t_{2}\right)\left(e^{u_{4}\left(t_{2}\right)-u_{2}\left(t_{2}\right)}-1\right)=0, \\
b_{2}\left(t_{3}\right) e^{u_{4}\left(t_{3}\right)-u_{3}\left(t_{3}\right)}-g_{2}\left(t_{3}\right)-d_{2}\left(t_{3}\right) e^{u_{3}\left(t_{3}\right)}=0,
\end{gathered}
$$


and

$$
g_{2}\left(t_{4}\right) e^{u_{3}\left(t_{4}\right)-u_{4}\left(t_{4}\right)}-D_{2}\left(t_{4}\right) e^{u_{4}\left(t_{4}\right)}+\lambda_{2}\left(t_{4}\right)\left(e^{u_{2}\left(t_{4}\right)-u_{4}\left(t_{4}\right)}-1\right)=0 .
$$

Thus we obtain

$$
\begin{gathered}
d_{1}\left(t_{1}\right) e^{2 u_{1}\left(t_{1}\right)}+g_{1}\left(t_{1}\right) e^{u_{1}\left(t_{1}\right)}=b_{1}\left(t_{1}\right) e^{u_{2}\left(t_{1}\right)} \\
D_{1}\left(t_{2}\right) e^{2 u_{2}\left(t_{2}\right)}+\lambda_{1}\left(t_{2}\right) e^{u_{2}\left(t_{2}\right)}=g_{1}\left(t_{2}\right) e^{u_{1}\left(t_{2}\right)}+\lambda_{1}\left(t_{2}\right) e^{u_{4}\left(t_{2}\right)} \\
d_{2}\left(t_{3}\right) e^{2 u_{3}\left(t_{3}\right)}+g_{2}\left(t_{3}\right) e^{u_{3}\left(t_{3}\right)}=b_{2}\left(t_{3}\right) e^{u_{4}\left(t_{3}\right)}
\end{gathered}
$$

and

$$
D_{2}\left(t_{4}\right) e^{2 u_{4}\left(t_{4}\right)}+\lambda_{2}\left(t_{4}\right) e^{u_{4}\left(t_{4}\right)}=g_{2}\left(t_{4}\right) e^{u_{3}\left(t_{4}\right)}+\lambda_{2}\left(t_{4}\right) e^{u_{2}\left(t_{4}\right)} .
$$

Equation (2.37) implies that

$$
D_{1}^{u} e^{2 u_{2}\left(t_{2}\right)}+\lambda_{1}^{u} e^{u_{2}\left(t_{2}\right)}>g_{1}^{l} e^{u_{1}\left(t_{1}\right)} .
$$

From (2.36) and (2.40), we have

$$
\begin{aligned}
b_{1}^{l} e^{u_{2}\left(t_{2}\right)}< & b_{1}\left(t_{1}\right) e^{u_{2}\left(t_{1}\right)}<d_{1}^{u} e^{2 u_{1}\left(t_{1}\right)}+g_{1}^{u} e^{u_{1}\left(t_{1}\right)} \\
& <\frac{g_{1}^{u}}{g_{1}^{l}}\left(D_{1}^{u} e^{2 u_{2}\left(t_{2}\right)}+\lambda_{1}^{u} e^{u_{2}\left(t_{2}\right)}\right)+\frac{d_{1}^{u}}{\left(g_{1}^{l}\right)^{2}}\left(D_{1}^{u} e^{2 u_{2}\left(t_{2}\right)}+\lambda_{1}^{u} e^{u_{2}\left(t_{2}\right)}\right)^{2},
\end{aligned}
$$

that is,

$$
\begin{aligned}
\left(g_{1}^{l}\right)^{2} b_{1}^{l} e^{u_{2}\left(t_{2}\right)} & <g_{1}^{u} g_{1}^{l} D_{1}^{u} e^{2 u_{2}\left(t_{2}\right)}+g_{1}^{u} g_{1}^{l} \lambda_{1}^{u} e^{u_{2}\left(t_{2}\right)} \\
& +d_{1}^{u}\left(D_{1}^{u}\right)^{2} e^{4 u_{2}\left(t_{2}\right)}+d_{1}^{u}\left(\lambda_{1}^{u}\right)^{2} e^{2 u_{2}\left(t_{2}\right)}+2 d_{1}^{u} D_{1}^{u} \lambda_{1}^{u} e^{3 u_{2}\left(t_{2}\right)}
\end{aligned}
$$

Thus

$$
\begin{gathered}
d_{1}^{u}\left(D_{1}^{u}\right)^{2} e^{3 u_{2}\left(t_{2}\right)}+2 d_{1}^{u} D_{1}^{u} \lambda_{1}^{u} e^{2 u_{2}\left(t_{2}\right)}+\left[g_{1}^{u} g_{1}^{l} D_{1}^{u}+d_{1}^{u}\left(\lambda_{1}^{u}\right)^{2}\right] e^{u_{2}\left(t_{2}\right)} \\
>\left(g_{1}^{l}\right)^{2} b_{1}^{l}-g_{1}^{u} g_{1}^{l} \lambda_{1}^{u}=g_{1}^{l}\left(g_{1}^{l} b_{1}^{l}-g_{1}^{u} \lambda_{1}^{u}\right)
\end{gathered}
$$

Let $e^{u_{2}\left(t_{2}\right)}=z$. Then there exist some positive constants $p_{i}(i=0,1,2)$ such that $z^{3}+p_{2} z^{2}+p_{1} z=p_{0}$. Let $F(z)=z^{3}+p_{2} z^{2}+p_{1} z-p_{0}$. Then $F(0)=-p_{0}<0$, $F(+\infty)=+\infty>0$. Hence there exists a point $\xi_{2} \in[0, \omega]$ such that $F\left(\xi_{2}\right)=0$. Thus from $z^{3}+p_{2} z^{2}+p_{1} z-p_{0}>0$, we have $\left(z-\xi_{2}\right)\left[z^{2}+\left(p_{2}+\xi_{2}\right) z+\frac{p_{0}}{\xi_{2}}\right]>0$. Therefore $z>\xi_{2}$, a.e., $e^{u_{2}\left(t_{2}\right)}>\xi_{2}$. From this, for $\forall t \in[0, \omega]$, we have

$$
e^{u_{2}(t)}>\xi_{2} \text {. }
$$

Using a parallel argument to (2.41), from (2.38) and (2.39), it follows that there exists a positive constant $\xi_{4}$ such that

$$
e^{u_{4}(t)}>\xi_{4}
$$

From (2.36), (2.38), (2.41) and (2.42), we have

$$
e^{u_{1}(t)}>\xi_{1}, \quad e^{u_{3}(t)}>\xi_{3},
$$

where $\xi_{1}$ and $\xi_{3}$ are two positive constants. 
From (2.25)-(2.27), it follows that there exist four points $\eta_{i} \in[0, \omega], i=1,2,3,4$, such that

$$
u_{i}\left(\eta_{i}\right)<\ln \frac{\rho_{i}}{\omega}, \quad i=1,2,3,4 .
$$

From (2.41)-(2.43), it follows that there exist four positive constants $\rho_{i}^{*}$ such that

$$
u_{i}(t)>-\rho_{i}^{*} .
$$

Since for $\forall t \in[0, \omega]$,

$$
u_{i}(t)=u_{i}\left(\eta_{i}\right)-\int_{\eta_{i}}^{t} u_{i}^{\prime}(s) d s, \quad i=1,2,3,4,
$$

from (2.28)-(2.31) and (2.44), we have

$$
u_{i}(t)<\ln \frac{\rho_{i}}{\omega}+\int_{0}^{\omega}\left|u_{i}^{\prime}(s)\right| d s<\left|\ln \frac{\rho_{i}}{\omega}\right|+\delta_{i}, \quad i=1,2,3,4 .
$$

From (2.45) and (2.46), we can obtain

$$
\left|u_{i}(t)\right| \leq \max \left\{\rho_{i}^{*},\left|\ln \frac{\rho_{i}}{\omega}\right|+\delta_{i}\right\} \stackrel{\text { def }}{=} R_{i}, \quad i=1,2,3,4 .
$$

Clearly, $R_{i}(i=1,2,3,4)$ are independent of $\lambda$. Denote $M=R_{1}+R_{2}+R_{3}+R_{4}+R_{0}$. Here $R_{0}$ is taken sufficiently large such that each solution $\left(\alpha^{*}, \beta^{*}, \gamma^{*}, \delta^{*}\right)^{T}$ of the system

$$
\left\{\begin{array}{l}
\bar{b}_{1} e^{\beta-\alpha}-\bar{g}_{1}-\bar{d}_{1} e^{\alpha}=0 \\
\bar{g}_{1} e^{\alpha-\beta}-\bar{D}_{1} e^{\beta}+\bar{\lambda}_{1}\left(e^{\delta-\beta}-1\right)=0, \\
\bar{b}_{2} e^{\delta-\gamma}-\bar{g}_{2}-\bar{d}_{2} e^{\gamma}=0 \\
\bar{g}_{2} e^{\gamma-\delta}-\bar{D}_{2} e^{\delta}+\bar{\lambda}_{2}\left(e^{\beta-\delta}-1\right)=0
\end{array}\right.
$$

satisfies $\left\|\left(\alpha^{*}, \beta^{*}, \gamma^{*}, \delta^{*}\right)^{T}\right\|=\left|\alpha^{*}\right|+\left|\beta^{*}\right|+\left|\gamma^{*}\right|+\left|\delta^{*}\right|<M$, provided that system (2.47) has a solution or a number of solutions and that

$$
\begin{aligned}
& \max \left\{\rho_{2}^{*},|\ln l|\right\}+\max \left\{\rho_{1}^{*}, \ln \sqrt{\frac{\bar{b}_{1} l}{\bar{d}_{1}}}\right\} \\
& +\max \left\{\rho_{3}^{*},\left|\frac{1}{2} \ln \frac{\bar{b}_{2}}{\bar{d}_{2}}\right|+\mid \ln \left(\frac{\bar{g}_{2}}{2 \bar{D}_{2}} \sqrt{\frac{\bar{b}_{2}}{\bar{d}_{2}}}+\sqrt{\frac{\bar{\lambda}_{2} l}{\bar{D}_{2}}}+\sqrt{\left.\left.\frac{\bar{D}_{1} \bar{g}_{1}}{2 \bar{D}_{2}} \sqrt{\frac{\bar{b}_{1}}{\bar{d}_{1}}}\right) \mid\right\}}\right.\right. \\
& +\max \left\{\rho_{4}^{*}, \mid \ln \left(\frac{\bar{g}_{2} \sqrt{\frac{\bar{b}_{2}}{d_{2}}}}{2 \bar{D}_{2}}+\sqrt{\frac{\bar{\lambda}_{2} l}{\bar{D}_{2}}}+\sqrt{\left.\left.\frac{\bar{D}_{1} \bar{g}_{1}}{2 \bar{D}_{2}} \sqrt{\frac{\bar{b}_{1}}{\bar{d}_{1}}}\right) \mid\right\}<M .}\right.\right.
\end{aligned}
$$

Here, $l$ is the only real root of equation

$$
\bar{D}_{1} x=\frac{\bar{g}_{1}}{2} \sqrt{\frac{\bar{b}_{1}}{\bar{d}_{1}}}+\sqrt{\bar{D}_{1} \bar{\lambda}_{1}}\left[\sqrt{\frac{\bar{g}_{2}}{2 \bar{D}_{2}} \sqrt{\frac{\bar{b}_{2}}{\bar{d}_{2}}}}+\sqrt[4]{\frac{\bar{\lambda}_{2}}{\bar{D}_{2}}} x^{\frac{1}{4}}+\sqrt[4]{\frac{\bar{D}_{1} \bar{g}_{1}}{2 \bar{D}_{2}} \sqrt{\frac{\bar{b}_{1}}{\bar{d}_{1}}}}\right]+\sqrt{\frac{\bar{D}_{1} \bar{g}_{1}}{2 \bar{D}_{2}} \sqrt{\frac{\bar{b}_{1}}{\bar{d}_{1}}}} .
$$

Now we take $\Omega=\left\{\left(u_{1}(t), u_{2}(t), u_{3}(t), u_{4}(t)\right)^{T} \in X:\left\|\left(u_{1}, u_{2}, u_{3}, u_{4}\right)^{T}\right\|<M\right\}$. This satisfies condition (i) of Lemma 2.1. When $\left(u_{1}, u_{2}, u_{3}, u_{4}\right)^{T} \in \partial \Omega \cap \operatorname{Ker} L=\partial \Omega \cap R^{4}$, 
$\left(u_{1}, u_{2}, u_{3}, u_{4}\right)^{T}$ is a constant vector in $R^{4}$ with $\left|u_{1}\right|+\left|u_{2}\right|+\left|u_{3}\right|+\left|u_{4}\right|=M$. If system (2.47) has a solution or a number of solutions, then

$$
Q N\left[\begin{array}{l}
u_{1} \\
u_{2} \\
u_{3} \\
u_{4}
\end{array}\right]=\left[\begin{array}{c}
\bar{b}_{1} e^{u_{2}-u_{1}}-\bar{g}_{1}-\bar{d}_{1} e^{u_{1}} \\
\bar{g}_{1} e^{u_{1}-u_{2}}-\bar{D}_{1} e^{u_{2}}+\bar{\lambda}_{1}\left(e^{u_{4}-u_{2}}-1\right) \\
\bar{b}_{2} e^{u_{4}-u_{3}}-\bar{g}_{2}-\bar{d}_{2} e^{u_{3}} \\
\bar{g}_{2} e^{u_{3}-u_{4}}-\bar{D}_{2} e^{u_{4}}+\bar{\lambda}_{2}\left(e^{u_{2}-u_{4}}-1\right)
\end{array}\right] \neq 0 .
$$

If system (2.47) does not have a solution, then naturally

$$
Q N\left[\begin{array}{l}
u_{1} \\
u_{2} \\
u_{3} \\
u_{4}
\end{array}\right] \neq\left[\begin{array}{l}
0 \\
0 \\
0 \\
0
\end{array}\right]
$$

This proves that condition (ii) of Lemma 2.1 is satisfied. Finally we will prove that condition (iii) of Lemma 2.1 is satisfied. To this end, we define $\phi: \operatorname{DomL} \times[0,1] \rightarrow X$ by

$$
\phi\left(u_{1}, u_{2}, u_{3}, u_{4}, \mu\right)=\left[\begin{array}{c}
\bar{b}_{1} e^{u_{2}-u_{1}}-\bar{d}_{1} e^{u_{1}} \\
\bar{g}_{1} e^{u_{1}-u_{2}}-\bar{D}_{1} e^{u_{2}} \\
\bar{b}_{2} e^{u_{4}-u_{3}}-\bar{d}_{2} e^{u_{3}} \\
\bar{g}_{2} e^{u_{3}-u_{4}}-\bar{D}_{2} e^{u_{4}}
\end{array}\right]+\mu\left[\begin{array}{c}
-\bar{g}_{1} \\
\bar{\lambda}_{1}\left(e^{u_{4}-u_{2}}-1\right) \\
-\bar{g}_{2} \\
\bar{\lambda}_{2}\left(e^{u_{2}-u_{4}}-1\right)
\end{array}\right],
$$

where $\mu \in[0,1]$ is a parameter. When $\left(u_{1}, u_{2}, u_{3}, u_{4}\right)^{T} \in \partial \Omega \cap \operatorname{Ker} L=\partial \Omega \cap R^{4}$, $\left(u_{1}, u_{2}, u_{3}, u_{4}\right)^{T}$ is a constant vector in $R^{4}$ with $\left|u_{1}\right|+\left|u_{2}\right|+\left|u_{3}\right|+\left|u_{4}\right|=M$. We will show that when $\left(u_{1}, u_{2}, u_{3}, u_{4}\right)^{T} \in \partial \Omega \cap \operatorname{Ker} L, \phi\left(u_{1}, u_{2}, u_{3}, u_{4}, \mu\right) \neq 0$. If the conclusion is not true, a.e., the constant vector $\left(u_{1}, u_{2}, u_{3}, u_{4}\right)^{T}$ with $\left|u_{1}\right|+\left|u_{2}\right|+\left|u_{3}\right|+\left|u_{4}\right|=M$ satisfies $\phi\left(u_{1}, u_{2}, u_{3}, u_{4}, \mu\right)=0$, and then from

$$
\left\{\begin{array}{l}
\bar{b}_{1} e^{u_{2}-u_{1}}-\bar{d}_{1} e^{u_{1}}-\mu \bar{g}_{1}=0, \\
\bar{g}_{1} e^{u_{1}-u_{2}}-\bar{D}_{1} e^{u_{2}}+\mu \bar{\lambda}_{1}\left(e^{u_{4}-u_{2}}-1\right)=0, \\
\bar{b}_{2} e^{u_{4}-u_{3}}-\bar{d}_{2} e^{u_{3}}-\mu \bar{g}_{2}=0, \\
\bar{g}_{2} e^{u_{3}-u_{4}}-\bar{D}_{2} e^{u_{4}}+\mu \bar{\lambda}_{2}\left(e^{u_{2}-u_{4}}-1\right)=0,
\end{array}\right.
$$

by following the argument of (2.44) and (2.45), we obtain

$$
\begin{gathered}
\left|u_{1}\right|<\max \left\{\rho_{1}^{*},\left|\ln \sqrt{\frac{\bar{b}_{1} l}{\bar{d}_{1}}}\right|\right\}, \\
\left|u_{2}\right|<\max \left\{\rho_{2}^{*},|\ln l|\right\} \\
\left|u_{3}\right|<\max \left\{\rho_{3}^{*},\left|\frac{1}{2} \ln \frac{\bar{b}_{2}}{\bar{d}_{2}}\right|+\left|\ln \left(\frac{\bar{g}_{2}}{2 \bar{D}_{2}} \sqrt{\frac{\bar{b}_{2}}{\bar{d}_{2}}}+\sqrt{\frac{\bar{\lambda}_{2} l}{\bar{D}_{2}}}+\sqrt{\frac{\bar{D}_{1} \bar{g}_{1}}{2 \bar{D}_{2}} \sqrt{\frac{\bar{b}_{1}}{\bar{d}_{1}}}}\right)\right|\right\}
\end{gathered}
$$

and

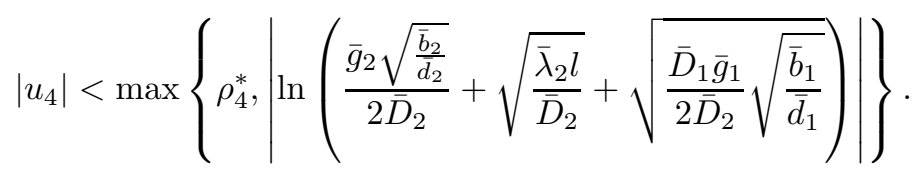


Thus

$$
\begin{aligned}
& \sum_{i=1}^{4}\left|u_{i}\right|<\max \left\{\rho_{2}^{*},|\ln l|\right\}+\max \left\{\rho_{1}^{*}, \ln \sqrt{\frac{\bar{b}_{1} l}{\bar{d}_{1}}}\right\}
\end{aligned}
$$

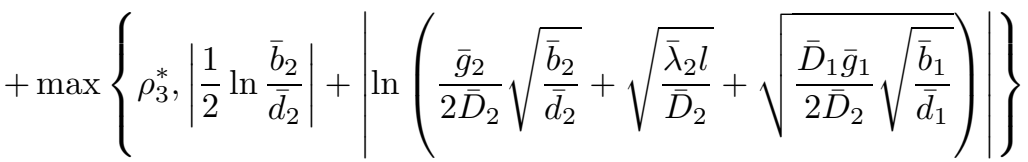

$$
\begin{aligned}
& +\max \left\{\rho_{4}^{*},\left|\ln \left(\frac{\bar{g}_{2} \sqrt{\frac{\bar{b}_{2}}{d_{2}}}}{2 \bar{D}_{2}}+\sqrt{\frac{\bar{\lambda}_{2} l}{\bar{D}_{2}}}+\sqrt{\frac{\bar{D}_{1} \bar{g}_{1}}{2 \bar{D}_{2}} \sqrt{\frac{\bar{b}_{1}}{\bar{d}_{1}}}}\right)\right|\right\}<M,
\end{aligned}
$$

which contradicts the fact that $\sum_{i=1}^{4}\left|u_{i}\right|=M$. Therefore when $\left(u_{1}, u_{2}, u_{3}, u_{4}\right)^{T} \in$ $\partial \Omega \cap \operatorname{Ker} L, \phi\left(u_{1}, u_{2}, u_{3}, u_{4}, \mu\right) \neq 0$. According to topological degree theory and by taking $J=I: \operatorname{Im} Q \rightarrow \operatorname{Ker} L,\left(u_{1}, u_{2}, u_{3}, u_{4}\right)^{T} \rightarrow\left(u_{1}, u_{2}, u_{3}, u_{4}\right)^{T}$, we have

$$
\begin{aligned}
& \operatorname{deg}\left(J Q N\left(u_{1}, u_{2}, u_{3}, u_{4}\right)^{T}, \Omega \cap \operatorname{Ker} L,(0,0,0,0)^{T}\right) \\
= & \operatorname{deg}\left(\phi\left(u_{1}, u_{2}, u_{3}, u_{4}, 1\right), \Omega \cap \operatorname{Ker} L,(0,0,0,0)^{T}\right) \\
= & \operatorname{deg}\left(\phi\left(u_{1}, u_{2}, u_{3}, u_{4}, 0\right), \Omega \cap \operatorname{Ker} L,(0,0,0,0)^{T}\right) \\
= & \operatorname{deg}\left(\left(\bar{b}_{1} e^{u_{2}-u_{1}}-\bar{d}_{1} e^{u_{1}}, \bar{g}_{1} e^{u_{1}-u_{2}}-\bar{D}_{1} e^{u_{2}}, \bar{b}_{2} e^{u_{4}-u_{3}}-\bar{d}_{2} e^{u_{3}},\right.\right. \\
& \left.\left.\bar{g}_{2} e^{u_{3}-u_{4}}-\bar{D}_{2} e^{u_{4}}\right)^{T}, \Omega \cap \operatorname{Ker} L,(0,0,0,0)^{T}\right) .
\end{aligned}
$$

In view of the fact that the system of algebraic equations

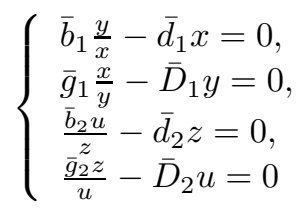

has a unique solution $\left(x^{*}, y^{*}, z^{*}, u^{*}\right)^{T}$ which satisfies $x^{*}>0, y^{*}>0, z^{*}>0$ and $u^{*}>0$, it follows that

$$
\begin{aligned}
& \operatorname{deg}\left(\left(\bar{b}_{1} e^{u_{2}-u_{1}}-\bar{d}_{1} e^{u_{1}}, \bar{g}_{1} e^{u_{1}-u_{2}}-\bar{D}_{1} e^{u_{2}}, \bar{b}_{2} e^{u_{4}-u_{3}}-\bar{d}_{2} e^{u_{3}},\right.\right. \\
& \left.\left.\bar{g}_{2} e^{u_{3}-u_{4}}-\bar{D}_{2} e^{u_{4}}\right)^{T}, \Omega \cap \operatorname{Ker} L,(0,0,0,0)^{T}\right) \\
& =\operatorname{sign}\left|\begin{array}{cccc}
-\frac{\bar{b}_{1} y^{*}}{x^{*}}-\bar{d}_{1} x^{*} & \frac{\bar{b}_{1} y^{*}}{x^{*}} & 0 & 0 \\
\frac{\bar{g}_{1} x^{*}}{y^{*}} & \frac{-\bar{g}_{1} x^{*}}{y^{*}}-\bar{D}_{1} y^{*} & 0 & 0 \\
0 & 0 & \frac{-\bar{b}_{2} u^{*}}{z^{*}}-\bar{d}_{2} z^{*} & \frac{\bar{b}_{2} u^{*}}{u^{*} z^{*}} \\
0 & 0 & \frac{-\bar{g}_{2} z^{*}}{u^{*}}-\bar{D}_{2} u^{*}
\end{array}\right| \\
& =\operatorname{sign}\left[\left(\frac{\bar{b}_{1} \bar{D}_{1}\left(y^{*}\right)^{2}}{x^{*}}+\frac{\bar{d}_{1} \bar{g}_{1}\left(x^{*}\right)^{2}}{y^{*}}+\bar{d}_{1} \bar{D}_{1} x^{*} y^{*}\right)\right. \\
& \left.\times\left(\frac{\bar{d}_{2} \bar{g}_{2}\left(z^{*}\right)^{2}}{u^{*}}+\bar{d}_{2} \bar{D}_{2} u^{*} z^{*}+\frac{\bar{b}_{2} \bar{D}_{2}\left(u^{*}\right)^{2}}{z^{*}}\right)\right] \\
& =1 \text {. }
\end{aligned}
$$


This completes the proof of condition (iii) of Lemma 2.1. Now $\Omega$ verifies all the requirements of Lemma 2.1 and then system (2.1) has at least one $\omega$-periodic solution. This completes the proof of Theorem 2.1.

Acknowledgments. The authors are grateful to the anonymous referee for his/her helpful comments and valuable suggestions.

\section{REFERENCES}

[1] W.G. Aiello and H.I. Freedman, A time-delay model of single-species growth with stage structure, Math. Biosci., 101(1990), 139-153. MR1071716 (91f:92030)

[2] W.G. Aiello, H.I. Freedman, and J. Wu, Analysis of a model representing stage-structured population growth with stage-dependent time delay, SIAM J. Appl. Math. 3(1992), 855-869. MR1163810 (93j:92025)

[3] Wengdi Wang and Lansun Chen, A predator-prey system with stage-structure for predator, Computers Math. Applic. 8(1997), 83-91. MF1448315 (98d:92019)

[4] R. Mahbuba and Lansun Chen, On the nonautonomous Lotka-Volterra competition system with diffusion, Diff. Equt. and Dyn. Syst., 2(1994), 243-253. MR1386272 (97h:92021)

[5] H.I. Freedman and J. Wu, Periodic solutions of single-species models with periodic delay, SIAM J. Math. Anal., 23:3(1992), 689-701. MR1158828 (93e:92012)

[6] E. Beretta and Y. Takeuchi, Global stability of single-delayed single-species diffusion Volterra models with continuous time delays, Bull. Math. Biol., 49:4(1987), 431-448. MR0908159 (88k:92058)

[7] R.E. Games and J.L. Mawhin, "Coincidence degree and Nonlinear Differential Equations", SpringerVerlag, Berlin, 1977. MF0637067 (58:30551)

[8] J.L. Mawhin, "Topological Degree Methods in Nonlinear Boundary Value Problems", CBMS Regional Conf. Ser. in Math., No. 40, Am. Math. Soc. Providence, 1979. MR 0525202 (80c:47055)

[9] Y. Li, Periodic solution of a neutral delay equation, J. Math. Appl., 24(1997), 11-21. MR1645495 (99j:34101)

[10] Y. Li, Periodic solutions of a periodic delay predator-prey systm, Proc. Amer. Math. Soc., 127(1999), 1331-1335. MR1646198|(99i:34101) 